\title{
Prises de bec chez les phalaropes
}

David Quéré(1) (david.quere@espci.fr), Manu Prakash(2), John W.M. Bush(3)

(1) Physique et Mécanique des Milieux Hétérogènes, ESPCI, 10 rue Vauquelin, 75005 Paris

(2) Center for Bits and Atoms, MIT, Cambridge, MA 02139, USA

(3) Department of Mathematics, MIT, Cambridge, MA 02139, USA

\section{Nous décrivons ici comment}

le phalarope, petit oiseau

limicole des bords

de l'Arctique, se nourrit

au goutte-à-goutte

en utilisant à la fois

la géométrie de son bec

et un mouvement

mandibulaire maîtrisé

à la perfection.

II nous donne ainsi

l'exemple d'une pince à

liquide, permettant à la fois

la prise et la manipulation

de gouttes, sans influence

du champ de la pesanteur.

Remerciements

Les auteurs remercient spécialement les relecteurs pour leurs excellentes suggestions.

\section{l'hydrotechnique phalarope}

Les phalaropes sont des petits oiseaux limicoles qui vivent sur les rivages américains ou asiatiques de l'Arctique. Ils ont été découverts au dix-huitième siècle par George Edwards puis décrits et nommés par Brisson "à cause de leurs pieds semblables à ceux de la foulque ", nous dit Buffon - phalaris en grec. On les connait d'abord pour leur partage inhabituel des tâches : la femelle, plus grosse, migre vers le Sud (la mer d'Oman) peu après la ponte, tandis que les mâles couvent les œufs et s'occupent des oisillons.

Mais leur mode d'alimentation, décrit par les ornithologues dans les années 90, est plus curieux encore [1-2]. Le bec du phalarope est court, deux à trois centimètres, et ce drôle d'oiseau fait d'abord venir son repas à la surface de l'eau : à la différence des canards, il ne plonge pas pour pêcher et il est donc contraint de trouver sa subsistance au voisinage immédiat de la surface. Sa stratégie est révolutionnaire (à proprement parler) : le phalarope fait en nageant des ronds dans l'eau, des cercles d'un diamètre de quelques dizaines de centimètres, à une vitesse de l'ordre de $50 \mathrm{~cm} / \mathrm{s}$. Comme le savent les amateurs de thé, on engendre ainsi des écoulements qui remontent de la profondeur vers la surface les feuilles dans la tasse de thé - et des petits crustacés ou du plancton pour le phalarope. Il n'y a donc plus qu'à se servir, ce que notre oiseau fait à coups de bec (fig. 1), des coups fréquents (2 par seconde, environ) dont il tire à chaque fois non pas directement une proie mais une goutte d'eau. Alors que la direction du bec pointe vers le bas, la goutte remonte jusqu'au gosier où le phalarope extrait le nutriment qui s'y trouve (un par goutte, en moyenne) et l'avale.

Et nous en arrivons à la question qui va nous intéresser, qui est la façon dont cette goutte avance, contre la pesanteur et malgré sa grande fluidité [3].

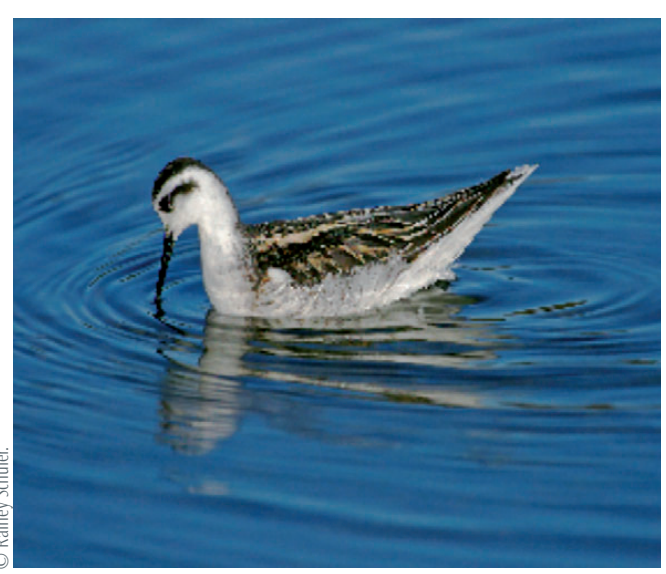

1. On voit ici un phalarope en action, au moment où il attrape un peu de liquide sous la surface de l'eau, pour se nourrir. La goutte qu'il extrait va remonter le long du bec sans que le phalarope l'aspire, mais sous l'effet conjoint de la géométrie du bec et d'un mouvement mandibulaire.

\section{Autopropulsion}

Avant de s'interroger sur l'origine du mouvement, on notera que la goutte doit, pour avancer, faire un pont entre le haut et le bas du bec. Ceci impose un angle maximum d'ouverture au bec. En effet, une goutte ne peut joindre deux plans que si la distance entre ces plans est au plus (de l'ordre de) la largeur de la goutte sinon, elle se casse en deux, et on perd la continuité du liquide d'un plan à l'autre. Les gouttes ont une taille de quelques millimètres, notée $a$. L'espacement au bout d'un bec de longueur $L_{b}$ ouvert d'un angle $\alpha$ valant $\alpha L_{b}$, la condition de stabilité s'écrit $\alpha<\alpha_{c} \sim a / L_{b}$, ce qui donne un angle critique $\alpha_{c}$ de quelques degrés seulement. Ouvrant plus largement son bec, l'infortuné phalarope perd son repas, tel le corbeau de la fable.

Comment la goutte remonte-t-elle alors le bec ? Une origine possible du mouvement est d'abord géométrique : la distance entre les parties supérieure et inférieure du bec décroît quand on approche du gosier, ce qui rend asymétrique la goutte qu'on y place. Or quand une interface liquide est courbée, le liquide sous cette surface est en surpression (dite de Laplace) ou en dépression, selon le signe de la courbure. Un liquide mouillant dans un tube capillaire sera ainsi tiré par le ménisque qui le borne : la courbure de ce ménisque engendre une dépression sous sa surface, qui agit bien 

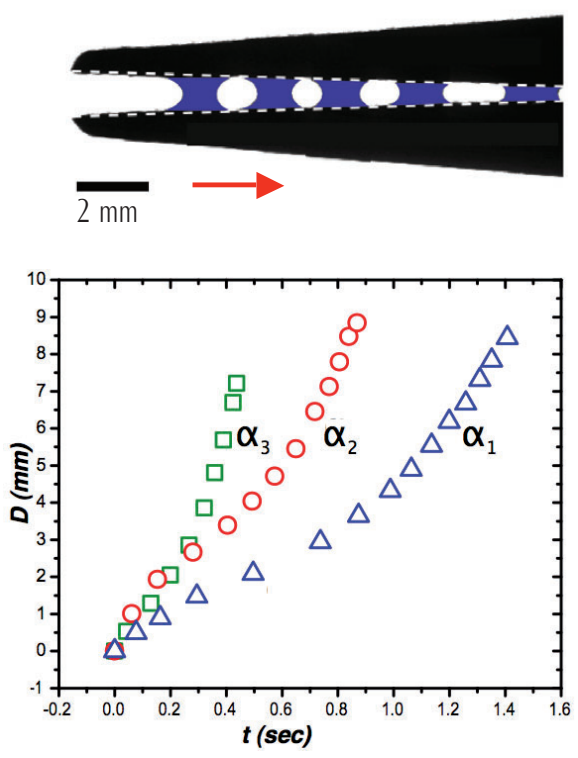

2. Positions successives d'une goutte d'huile silicone dans un bec artificiel horizontal, en fonction du temps. La flèche indique la direction du mouvement, et l'échelle 2 millimètres. On voit sur le graphe que la goutte avance à une vitesse constante d'autant plus grande que le bec est plus ouvert, avant d'accélérer dans la région la plus confinée du bec. Les angles $\alpha_{1}, \alpha_{2}$ et $\alpha_{3}$ valent $1,9^{\circ}, 2,8^{\circ}$ et $4,2^{\circ}$.
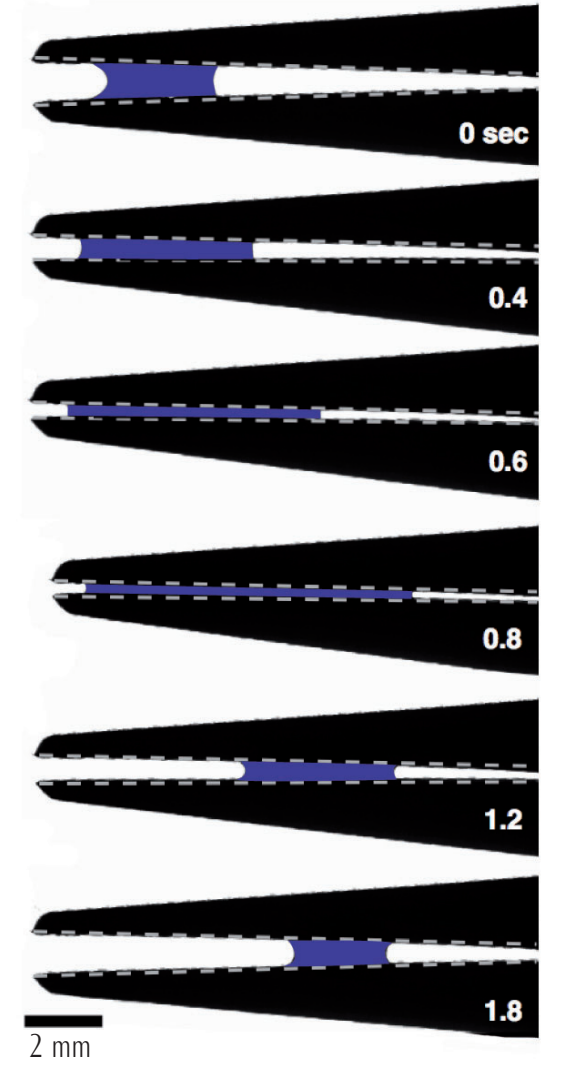

3. Progression d'une goutte d'eau dans un bec artificiel: le mouillage n'est que partiel, et la goutte avance parce qu'on ferme et ouvre cycliquement le bec. La vitesse moyenne dépend à la fois des angles extrêmes du mouvement mandibulaire et de la fréquence de ce mouvement. $0 \mathrm{n}$ a indiqué le temps à droite, en secondes, et l'échelle en bas à gauche représente $2 \mathrm{~mm}$. $\gg>$

comme un aspirateur pour le liquide. Si le liquide mouille le bec, ces deux ménisques n'ont pas la même courbure et l'aspiration différentielle qui en résulte propulse la goutte vers le côté le plus étroit, qui est celui du gosier (fig. 2).

À partir d'un bec artificiel comparable à celui du phalarope, nous avons caractérisé le mouvement de gouttes d'huile dans cet environnement asymétrique : une goutte placée au bout d'un de ces becs horizontaux progresse d'elle-même jusqu'à l'autre extrémité, à vitesse constante, sauf en fin de course où elle accélère (fig. 2). On devine qu'équilibrer la force motrice de Laplace par une résistance visqueuse peut conduire à une vitesse de progression constante. En revanche, l'accélération dans la région la plus confinée est inattendue, la résistance visqueuse augmentant fortement dans cette zone. Le lecteur curieux pourra se reporter à l'encadré (p. 13) pour trouver des explications plausibles à ces différentes observations.

\section{Nécessité d'un mouvement mandibulaire}

Il se trouve que le phalarope ne cuisine pas à l'huile, mais à l'eau. Or une goutte d'eau placée dans notre bec artificiel aura un comportement bien différent : elle reste bloquée là où on l'a mise. La (faible) force capillaire qui la pousse vers le gosier est (largement) contrebalancée par la force d'accrochage du liquide sur les défauts du solide. Tant qu'on est en mouillage complet (avec l'huile), des films moléculaires s'échappent de la goutte et " effacent " les hétérogénéités ; mais, avec l'eau, le mouillage n'est plus que partiel, et les défauts hydrophiles retiennent l'arrière de la goutte tandis que l'avant bute sur des zones non mouillantes. Ce phénomène nous est familier : c'est lui qui empêche les gouttelettes de pluie de dévaler sur les vitres, en dépit de la gravité. On voit bien de profil que ces gouttes sont plus bombées à l'aval qu'en amont, à cause de l'accrochage du liquide sur les défauts du solide. Dans le bec, l'angle de contact $\theta_{1}$ "à l'avant " de la goutte sera de la même manière plus grand que celui «à l'arrière ", noté $\theta_{2}$, ce qui permet aux courbures des ménisques d'encaisser la force capillaire qui tire la goutte d'eau vers le gosier. Il suffit que la différence $\theta_{2}-\theta_{1}$ soit de l'ordre de $\alpha$ pour que la goutte résiste. Or cette différence peut être communément de quelques dizaines de degrés sur des matériaux ordinaires, bien plus grande que les quelques degrés d'ouverture du bec, ce qui permet de comprendre pourquoi la goutte reste bloquée sur les défauts du solide.

La parade du phalarope, facile à imiter avec un bec artificiel, est d'imposer un mouvement mandibulaire qui alternativement écrase et relâche la goutte (fig. 3). On observe que le liquide avance alors de manière incrémentale, à chaque cycle du bec. L'angle $\alpha$ est, à présent, fonction du temps, partant d'une valeur maximale $\alpha_{M}$ pour décroître à un minimum $\alpha_{\mathrm{m}}$ avant de remonter à $\alpha_{\mathrm{M}}$, et ainsi de suite, à une fréquence d'une dizaine de Hertz. Cette fréquence élevée permet une progression rapide du liquide, à une vitesse de l'ordre du mètre par seconde. Mais nous allons surtout discuter comment les valeurs $\alpha_{M}$ et $\alpha_{m}$ conditionnent l'efficacité du processus.

\section{Optimisation mandibulaire}

Il est instructif de raisonner d'abord sur une goutte placée entre deux plans parallèles que l'on approche, puis que l'on éloigne l'un de l'autre. Tant que l'amplitude de l'oscillation est faible, la ligne de contact reste piégée sur les défauts ; mais au-delà d'un seuil, le liquide se décroche et parcourt une distance d'autant plus grande que l'oscillation est ample, sans toutefois que son centre de gravité ne progresse [4]. Dans cette expérience, le décrochage se produit quand l'angle de contact dépasse sa plus grande valeur statique possible, que l'on appelle l'angle d'avancée $\theta_{\mathrm{a}}$. De même, quand on écarte les plans l'un de l'autre, la goutte ne se rétracte que si l'angle devient inférieur à sa plus petite valeur statique, l'angle de reculée $\theta_{\mathrm{r}}$.

Si les plans ne sont plus parallèles, les valeurs extrêmes $\alpha_{M}$ et $\alpha_{m}$ définissent l'amplitude de l'oscillation. Le liquide ne bougera que si la quantité $\alpha_{M}-\alpha_{m}$ est assez grande, mais le point nouveau est que cela s'accompagne d'un déplacement de la goutte vers la région la plus confinée, c'est-à-dire le gosier de l'animal (fig. 3). Comment expliquer ce mouvement ? Nous schématisons ce qui se passe sur la figure 4. Quand le bec se ferme, les angles $\theta_{1}$ à l'avant et $\theta_{2}$ à l'arrière(1) augmentent comme précédemment, mais en partant d'une valeur différente : à cause de l'asymétrie du bec, on a initialement $\theta_{1}>\theta_{2}$, comme on l'a souligné plus haut. L'angle $\theta_{\mathrm{a}}$ pour lequel le liquide se met en mouvement sera donc atteint par l'avant de la goutte avant de l'être par l'arrière : l'avant seul progresse dans un premier temps. Si l'oiseau continue à fermer son bec, l'arrière à son tour se décroche des défauts du solide, 
mais en partant dans la direction opposée. Et quand le bec s'ouvre, c'est le contraire : l'arrière de la goutte va vers le gosier quand l'angle $\theta_{2}$ atteint la valeur $\theta_{\mathrm{r}}$, et l'avant ne recule que si le bec continue de s'ouvrir. On voit ainsi qu'un mouvement incrémental va se produire à chaque demi-cycle, dans le sens désiré.

Outre l'origine du mouvement, ces raisonnements permettent de comprendre que si le phalarope maitrise finement les angles extrêmes $\alpha_{M}$ et $\alpha_{m}$, il pourra ne propulser que l'avant de la goutte quand il ferme le bec (en laissant l'arrière bloqué), et que l'arrière quand il l'ouvre. Pour préciser ces phénomènes, nous avons mesuré le nombre de cycles nécessaires pour qu'une goutte remonte l'ensemble d'un bec artificiel (dont le mouillage se compare à celui du bec réel). On voit sur la figure 5 qu'il existe en effet une fenêtre étroite d'angles où le mouvement est optimisé : il suffit alors de trois cycles pour qu'une goutte millimétrique parcourre les deux centimètres du bec artificiel. Ce chiffre correspond aux observations faites par les ornithologues sur les phalaropes,

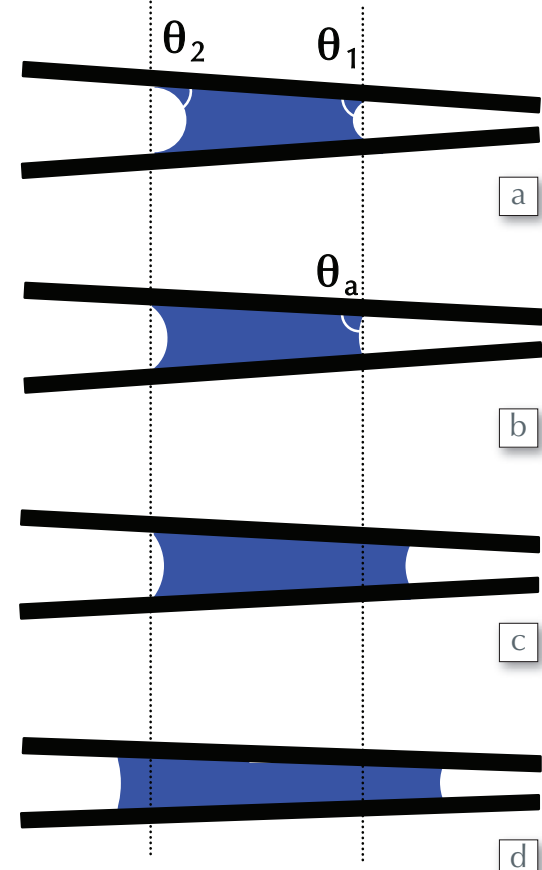

4. Schéma du mouvement d'une goutte en mouillage partiel dans un bec en fonction de l'angle d'ouverture. (a) La goutte reste bloquée à cause de l'accrochage de la ligne sur les défauts du solide, et résiste ainsi à la force capillaire qui tend à la propulser vers le gosier, ce qui se manifeste par deux angles de mouillage différents $\theta_{1}$ et $\theta_{2}$ à l'avant et à l'arrière, avec $\theta_{1}>\theta_{2}$. (b) Quand le phalarope ferme son bec, ces deux angles augmentent, mais c'est l'angle à l'avant $\theta_{1}$ qui atteint le premier la valeur $\theta_{a}$ au-delà de laquelle il y a mouvement (c). (d) Si le phalarope continue de fermer son bec, l'angle $\theta_{2}$ peut à son tour atteindre $\theta_{a}$, ce qui déclenche le recul de l'arrière de la goutte.

\section{Lois d'échelle pour des gouttes automotrices}

Il existe de nombreux dispositifs qui permettent le déplacement spontané d'une goutte posée sur un solide [6]. Dans tous les cas, le mouvement se fonde sur une asymétrie, qui peut être due au mouillage, à la température ou à la géométrie, comme dans le bec de phalarope où l'avant et l'arrière de la goutte ne voient pas le même degré de confinement. Si le bec est horizontal et le liquide mouillant (situation qui n'est pas celle du phalarope, mais qui est utile à caractériser), la goutte est aspirée vers le côté le plus étroit.

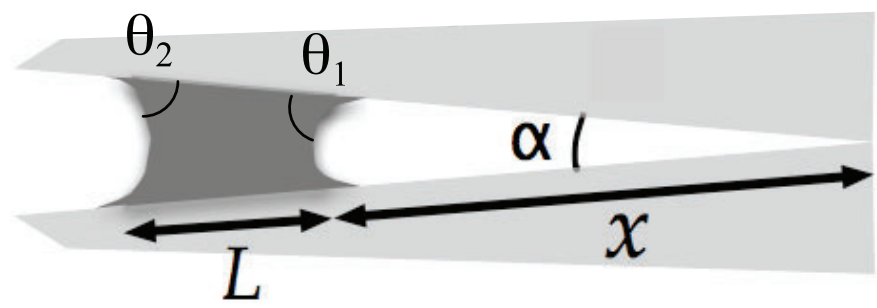

Goutte mouillante de longueur $L$ dans un bec ou dans un cône, à une distance $x$ du sommet.

Pour un liquide de tension superficielle $\gamma$, la traversée d'une interface courbée d'un rayon $R$ s'accompagne d'un saut de pression de Laplace $\gamma / R$, négatif pour des interfaces tournées vers l'air comme sur la figure (la pression est plus élevée du côté concave). Raisonnons pour des raisons de simplicité sur un cône. Les dépressions de Laplace à l'avant et à l'arrière d'une goutte mouillante $\left(\theta_{1}=\theta_{2}=0\right)$ s'écrivent en ordre de grandeur et en valeur absolue $\gamma / \alpha x$ et $\gamma / \alpha(x+L)$. Elles diffèrent donc de $\gamma L / \alpha x^{2}$ si la longueur de la goutte $L$ est petite devant la distance $x$ à la pointe. Intégrée sur la surface des ménisques $\left(d^{\prime}\right.$ ordre $\left.\alpha^{2} x^{2}\right)$, ceci donne une force $\alpha \gamma L$ qui tire la goutte vers la droite, sur la figure, et qui a la " bonne » propriété de disparaître si $\alpha=0$ : un pont liquide entre deux plans parallèles ne bouge pas.

Quand la goutte avance, cette force capillaire est contrebalancée par une force visqueuse, qui sera souvent une friction " de Stokes » : un objet de taille $L$ oppose au mouvement une force $\eta L V$, en notant $\eta$ la viscosité du liquide et $V$ sa vitesse. De cet équilibre de forces, on déduit une formule remarquablement simple pour la vitesse $: V \sim \alpha \gamma / \eta$.

Cette vitesse est constante le long de la course, alors que la longueur $L$ de l'index liquide augmente avec le temps, à cause de la géométrie. Elle apparait être le produit de l'angle $\alpha$ du bec, qui est petit (de l'ordre de 0,1 rd), par la vitesse « viscocapillaire » $\gamma / \eta$ du liquide, une quantité souvent grande : pour une huile qui a 20 fois la viscosité de l'eau et une tension de surface de $20 \mathrm{mN} / \mathrm{m}$, la quantité $\gamma / \eta$ vaut $1 \mathrm{~m} / \mathrm{s}$. On attend donc des vitesses $V$ de l'ordre de la dizaine de centimètres par seconde, et qui augmentent linéairement avec l'angle $\alpha$ (fig. 2).

Au-delà de cette phase de vitesse constante, on observe sur la figure 2 une accélération quand l'huile entre dans la région la plus confinée. Ce comportement est paradoxal : à cause de leur viscosité, les liquides ont en général tendance à avancer d'autant plus lentement qu'ils sont confinés. Or le frottement envisagé ci-dessus n'est pas le seul possible : près de la frontière d'une goutte, au voisinage de la ligne de contact, il existe une friction spéciale liée au fait que l'épaisseur de liquide s'annule à cet endroit. Cette friction est élevée, comme on le voit lorsque des gouttes dévalent sur une vitre : quoique ce soit de l'eau sur une pente forte, la vitesse reste modeste (comparée à celle d'une rivière, par exemple), à cause de cette forte friction de ligne. Or, dans le bec, le périmètre de la ligne diminue quand la goutte progresse ; en outre, la goutte s'allonge, ce qui rend cette friction de ligne de plus en plus petite, comparée à la force capillaire qui, elle, augmente avec $L$ : la goutte va donc accélérer.

Dans le cas du phalarope, la dynamique est très différente : I'eau ne mouille pas complètement le bec et s'accroche à ses défauts de surface, ce qui l'empêche d'avancer d'elle-même. Ce sont les mouvements mandibulaires qui induisent la propulsion du liquide, et la fréquence élevée du cycle de vibration qui la rend si rapide (autour du mètre par seconde). L'objet de cet article a été de montrer comment la conjonction du confinement (qui permet de capturer des gouttes) et de sa variation (qui permet de les propulser) engendre de véritables " pinces à gouttes ", dont nous espérons nous inspirer pour bien des applications.

(1) Ces angles, qui dépendent de l'histoire du système, sont compris entre $\theta_{\mathrm{r}}$ et $\theta_{\mathrm{a}}$ quand la goutte est à l'arrêt, et ils sont au-delà ou en deçà de ces valeurs quand il y a mouvement. 
5. Nombre de cycles mandibulaires pour qu'une goutte de 2 millimètres remonte les deux centimètres d'un bec artificiel. On constate l'existence d'une fenêtre étroite où le mouvement est optimisé : alors trois cycles suffisent pour remonter le bec, au lieu d'une dizaine si l'on ne règle pas avec soin les angles extrêmes d'ouverture et de fermeture $\alpha_{M}$ et $\alpha_{m}$ (exprimés en degrés). On a aussi noté les zones de non fonctionnement : une non physique $\left(\alpha_{m}\right.$ est par définition plus grand que $\alpha_{m}$ ), une de piégeage (où la ligne de contact ne bouge pas), une de détachement (au-dessus de l'angle $\alpha_{c}$, la goutte n'est plus coincée), et une d'étalement (à trop fermer le bec, on étale la goutte jusqu'au gosier)

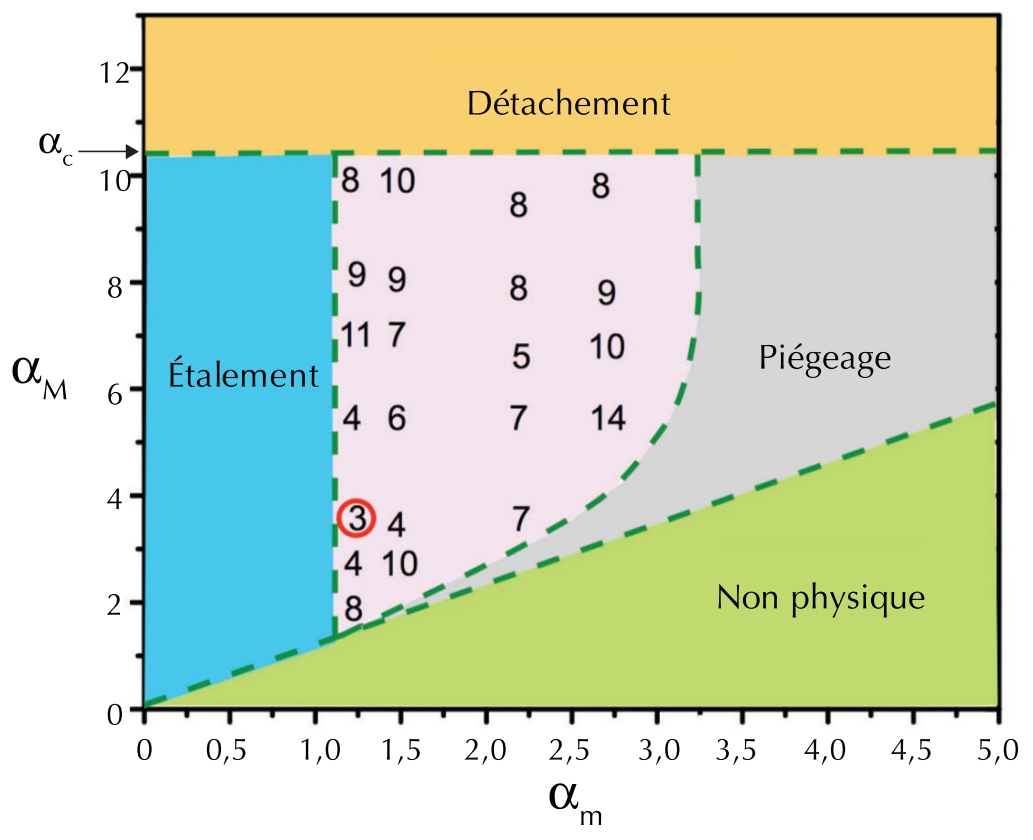

\section{Références}

1 M.A. Rubega, B.S. Obst, Auk 110 (1993) 169.

2 • S.M. Estrella, J.A. Masero, A. Perez-Hurtado, Auk 124 (2007) 1244

3 - M. Prakash, D. Quéré, J.W.M. Bush, Science $320(2008) 931$.

$4 \cdot X$. Noblin, A. Buquin, F. Brochard-Wyart, Eur. Phys. J. E 14 (2004) 395

5 •.W.M. Bush, D. Hu, M. Prakash, Adv. Insect Physiol. 34 (2008) 117.

6• J. Bico, D. Quéré, J. Fluid Mech. 467 (2002) 101. $\gg>$

dont le goutte-à-goutte est le seul mode d'alimentation. Il est frappant de relever que le mouvement mandibulaire n'est plus optimisé pour les volatiles qui n'utilisent qu'occasionnellement ce mode : ces oiseaux ont, eux, besoin de 7 ou 8 cycles pour conduire la goutte jusqu'à leur gosier ! De façon remarquable, la propulsion mandibulaire s'appuie donc sur les défauts de mouillage du bec. Mais ceux-ci offrent un autre avantage, tout aussi crucial : ils empêchent le liquide de retomber dans le champ de la gravité. Le bec du phalarope est presque toujours vertical (fig. 1), et le scénario décrit ici s'applique quelle que soit la direction du bec. A contrario, ceci souligne l'extrême sensibilité du phalarope à la pollution : en présence d'huile ou de détergent, l'eau peut devenir mouillante pour le bec (comme l'huile l'était) ; la pesanteur retrouve alors ses droits, empêchant le liquide d'être hissé jusqu'au gosier. De ce point de vue, les phalaropes se rapprochent des animaux tributaires de la tension de surface, comme les gerris qui se déplacent à la surface de l'eau. Dans les deux cas, une pollution de surface peut être fatale, et d'autant plus pernicieuse que de petites quantités de contaminants suffisent à la provoquer. On voit là certains des risques qu'il y a à mener une vie trop superficielle! 